\title{
On some Hall polynomials over a quiver of type $\tilde{\mathrm{D}}_{4}$
}

\author{
Csaba Szántó \\ Babeş-Bolyai University, Cluj-Napoca \\ Faculty of Mathematics and \\ Computer Science \\ Str. Mihail Kogalniceanu nr. 1 \\ R0-400084 Cluj-Napoca \\ Romania \\ email: szanto.cs@gmail.com
}

István Szöllősi

Babeş-Bolyai University, Cluj-Napoca

Faculty of Mathematics and

Computer Science

Str. Mihail Kogalniceanu nr. 1

R0-400084 Cluj-Napoca

Romania

email: szollosi@gmail.com

\begin{abstract}
Let $k$ be an arbitrary field and $Q$ a tame quiver of type $\tilde{D}_{4}$. Consider the path algebra $\mathrm{kQ}$ and the category of finite dimensional right modules mod- $k Q$. We determine the Hall polynomials $F_{x y}^{z}$ associated to indecomposable modules of defect $\partial z=-2, \partial x=\partial y=-1$ or dually $\partial z=2, \partial x=\partial y=1$.
\end{abstract}

\section{Introduction}

Classical Hall algebras associated with discrete valuation rings were introduced by Steinitz and Hall to provide an algebraic approach to the classical combinatorics of partitions. The multiplication is given by Hall polynomials which play an important role in the representation theory of the symmetric groups and the general linear groups. In 1990 Ringel defined Hall algebras for a large class of rings, namely finitary rings, including in particular path algebras of quivers over finite fields. Far reaching analogues of the classical ones, these Ringel-Hall algebras provided a new approach to the study of quantum groups using the representation theory of finite dimensional algebras. They 
can also be used successfully in the theory of cluster algebras or to investigate the structure of the module category.

In case of Ringel-Hall algebras corresponding to Dynkin quivers and tame quivers we know due to Ringel and Hubery, that the structure constants of the multiplication are again polynomials in the number of elements of the base field. These are the generalized Hall polynomials. If we are looking at Hall polynomials associated to indecomposable modules, the classical ones are just 0 or 1 , the generalized ones in the Dynkin case are also known and have degree up to 5 , however we do not have too much information about the generalized ones in the tame case. The first lists of particular tame Hall polynomials were given by the authors in [6] and in [7]. In [6] we presented all the tame Hall polynomials associated to indecomposable modules of defect $-1,0,1$. In [7] we listed the tame Hall polynomials corresponding to exact sequences of the form $\mathrm{O} \rightarrow \mathrm{P} \rightarrow \mathrm{R} \rightarrow \mathrm{I} \rightarrow 0$, where $\mathrm{P}$ is a preprojective, I a preinjective indecomposable and $R$ is a homogeneous module of dimension $\delta$ (the minimal radical vector of the tame quiver).

In this paper we restrict ourselves to the tame quiver of type $\tilde{\mathrm{D}}_{4}$ and determine all the tame Hall polynomials $\mathrm{F}_{x y}^{z}$ associated to indecomposable modules of defect $\partial z=-2, \partial x=\partial y=-1$ or dually $\partial z=2, \partial x=\partial y=1$.

\section{Preliminaries}

We begin with some facts related to representations of tame quivers. For a detailed description we refer to $[1,2,3]$.

Let $Q=\left(Q_{0}, Q_{1}\right)$ be a tame quiver without oriented cycles. Suppose that the vertex set $Q_{0}$ has $n$ elements and for an arrow $\alpha \in Q_{1}$ we denote by $t(\alpha), h(\alpha) \in Q_{0}$ the tail and head of $\alpha$. The Euler form of $Q$ is a bilinear form on $\mathbb{Z} Q_{0} \cong \mathbb{Z}^{n}$ given by $\langle x, y\rangle=\sum_{i \in Q_{0}} x_{i} y_{i}-\sum_{\alpha \in Q_{1}} x_{t(\alpha)} y_{h(\alpha)}$. Its quadratic form $\mathrm{q}_{\mathrm{Q}}$ (called Tits form) is independent from the orientation of $\mathrm{Q}$ and in the tame case it is positive semidefinite with radical $\mathbb{Z} \delta$, where $\delta$ is a minimal positive imaginary root of the corresponding Kac-Moody root system (which is also the minimal radical vector of the Tits form). The defect of $x \in \mathbb{Z} \mathrm{Q}_{0}$ is then $\partial x=\langle\delta, x\rangle$.

Let $k$ be a field. The category mod-kQ will be identified with the category rep- $k Q$ of the finite dimensional $k$-representations of the quiver. We will denote by $[M]$ the isomorphism class of the module $M$, by $\alpha_{M}$ the number of its automorphisms, by $\operatorname{dim} M \in \mathbb{Q}_{0} \cong \mathbb{Z}^{\mathfrak{n}}$ its dimension vector and by $\partial \mathrm{M}=$ 
$\partial(\operatorname{dim} M)$ its defect. Using the Euler form one has for $X, Y \in \bmod -k Q$

$$
\langle\underline{\operatorname{dim}} X, \underline{\operatorname{dim}} Y\rangle=\operatorname{dim}_{k} \operatorname{Hom}(X, Y)-\operatorname{dim}_{k} \operatorname{Ext}^{1}(X, Y) \text {. }
$$

For $\operatorname{dim}_{k} \operatorname{Hom}(X, Y)$ we will use the notation $(X, Y)$.

The indecomposable modules in mod-kQ are of three types: preprojectives (having negative defect), preinjectives (having positive defect) and regulars (having zero defect).

For $\mathrm{P}$ preprojective (i.e. with all its indecomposable components preprojective), I preinjective and $R$ regular module we have $\operatorname{Hom}(R, P)=\operatorname{Hom}(I, P)=$ $\operatorname{Hom}(I, R)=\operatorname{Ext}^{1}(P, R)=\operatorname{Ext}^{1}(P, I)=\operatorname{Ext}^{1}(R, I)=0$. It follows that the submodules of a preprojective module are always preprojective, preinjectives can project only on preinjectives, a submodule of a regular module cannot have preinjective components and a regular cannot project on preprojectives. Preprojective and preinjective indecomposables are exceptional (i.e. their endomorphism space is one dimensional and they have no self extensions) and are uniquely determined up to isomorphism by their dimension vector, which is a positive real root of the root system of Q. Note also that the possible defects of a preprojective indecomposable are -1 in the $\tilde{A}_{n}$ case, $-1,-2$ in the $\tilde{D}_{n}$ case, $-1,-2,-3$ in the $\tilde{E}_{6}$ case, $-1,-2,-3,-4$ in the $\tilde{E}_{7}$ case and $-1,-2,-3,-4,-5,-6$ in the $\tilde{\mathrm{E}}_{8}$ case.

The category of regular modules is an abelian, exact subcategory which decomposes into a direct sum of serial categories with Auslander-Reiten quiver of the form $Z \mathbb{A}_{\infty} / \mathrm{m}$, called tubes of rank $\mathrm{m}$. These tubes are indexed by the points of the projective line $\mathbb{P}_{k}^{1}$, the degree of a point $a \in \mathbb{P}_{k}^{1}$ being denoted by deg a. A tube of rank 1 is called homogeneous, otherwise it is called nonhomogeneous. We have at most 3 non-homogeneous tubes indexed by points a of degree $\operatorname{deg} a=1$. All the other tubes are homogeneous. We assume that the non-homogeneous tubes are labelled by some subset of $\{0,1, \infty\}$, whereas the homogeneous tubes are labelled by the closed points of the scheme $\mathbb{H}_{\mathrm{k}}=\mathbb{H}_{\mathbb{Z}} \otimes \mathrm{k}$ for some open integral subscheme $\mathbb{H}_{\mathbb{Z}} \subset \mathbb{P}_{\mathbb{Z}}^{1}$. Let $\mathbb{X}_{k} \subseteq \mathbb{H}_{k}$ be the set of points of degree 1 . The indecomposables on a homogeneous tube labelled by $a \in \mathbb{H}_{k}$ are denoted by $R^{k}(1, a) \subset R^{k}(2, a) \subset \ldots$ For a partition $\lambda=\left(\lambda_{1}, \ldots, \lambda_{n}\right)$ let $R^{k}(\lambda, a)=R^{k}\left(\lambda_{1}, a\right) \oplus \cdots \oplus R^{k}\left(\lambda_{n}, a\right)$. Note that the homogeneous modules of dimension $\delta$ are up to isomorphism $R^{k}(1, a)$, with $a \in \mathbb{X}_{k}$. For simplicity we will denote them by $R^{k}(a)$. Note that $\operatorname{dim}_{k} \operatorname{End}\left(R^{k}(a)\right)=1$.

A module without homogeneous regular components can be described combinatorially, field independently, using a system of positive real roots together with the dimension of quasi-socles for the non-homogeneous regular components of dimension $t \delta$. We denote this system by $\mu$ and let $M(\mu, k)$ be the cor- 
responding (up to isomorphism) unique module in mod-kQ. A Segre symbol is a multiset $\sigma=\left\{\left(\lambda^{1}, d_{1}\right), \ldots,\left(\lambda^{r}, d_{r}\right)\right\}$, where $\lambda^{i}$ are partitions and $d_{i} \in \mathbb{N}^{*}$. It will describe the homogeneous regular components of the module. Using the definitions above, a decomposition symbol is pair $\alpha=(\mu, \sigma)$. Given a decomposition symbol $\alpha=(\mu, \sigma)$ and a field $k$, we define the decomposition class $S(\alpha, k)$ to be the set of isomorphism classes of modules of the form $M(\mu, k) \oplus R$, where $R=R^{k}\left(\lambda^{1}, a_{1}\right) \oplus \cdots \oplus R^{k}\left(\lambda^{r}, a_{r}\right)$ for some distinct points $a_{1}, \ldots a_{r} \in \mathbb{H}_{k}$ such that $\operatorname{deg} a_{i}=d_{i}$. We also mention that for a decomposition symbol $\alpha$ the polynomial $n_{\alpha}(q)=|S(\alpha, k)|$ is strictly increasing in $q>1$.

Note that for $k$ finite with $q$ elements $\left|\mathbb{X}_{k}\right|=q+1$, $q$ or $q-1$ in the $\tilde{A}_{n}$ case and $q-2$ for other tame quivers. So if $k$ has 2 elements and the quiver is not of $\tilde{A}_{n}$ type there are no homogeneous modules of dimension $\delta$.

For simplicity denote by $x$ the decomposition symbol corresponding to a preprojective (preinjective) indecomposable given by the root $x$. Also denote by $\delta$ the symbol corresponding to homogeneous modules of dimension $\delta$.

We mention next some needed facts about Ringel-Hall algebras. Suppose that $k$ is finite. We consider the rational Ringel-Hall algebra $\mathcal{H}(\mathrm{kQ})$ of the algebra $k Q$. Its $\mathbb{Q}$-basis is formed by the isomorphism classes $[M]$ from mod-kQ and the multiplication is defined by $\left[N_{1}\right]\left[N_{2}\right]=\sum_{[M]} F_{N_{1} N_{2}}^{M}[M]$. The structure constants $F_{N_{1} N_{2}}^{M}=\left|\left\{U \subseteq M \mid U \cong N_{2}, M / U \cong N_{1}\right\}\right|$ are called Ringel-Hall numbers. The associativity of the Ringel-Hall algebra follows from the equality $\sum_{[\mathrm{N}]} \mathrm{F}_{\mathrm{N}_{1} \mathrm{~N}}^{\mathrm{M}} \mathrm{F}_{\mathrm{N}_{2} \mathrm{~N}_{3}}^{\mathrm{N}}=\sum_{[\mathrm{N}]} \mathrm{F}_{\mathrm{N}_{1} \mathrm{~N}_{2}}^{\mathrm{N}} \mathrm{F}_{\mathrm{NN}_{3}}^{\mathrm{M}}$.

Hubery proved the existence of generalized Hall polynomials in tame cases with respect to the decomposition classes.

Theorem 1 ([4]) Given decomposition symbols $\alpha, \beta$ and $\gamma$, there exists a rational polynomial $\mathrm{F}_{\alpha \beta}^{\gamma}$ such that for any finite field $\mathrm{k}$ with $|\mathrm{k}|=\mathrm{q}$,

$$
\mathrm{F}_{\alpha \beta}^{\gamma}(\mathrm{q})=\sum_{\substack{A \in S(\alpha, k) \\ \mathrm{B} \in \mathrm{S}(\beta, \mathrm{k})}} \mathrm{F}_{\mathrm{AB}}^{\mathrm{C}} \quad \text { for all } \mathrm{C} \in \mathrm{S}(\gamma, \mathrm{k})
$$

and moreover

$$
\begin{aligned}
& \mathrm{n}_{\gamma}(\mathrm{q}) \mathrm{F}_{\alpha \beta}^{\gamma}(\mathrm{q})=\mathrm{n}_{\alpha}(\mathrm{q}) \sum_{\substack{\mathrm{B} \in S(\beta, k) \\
\mathrm{C} \in S(\gamma, k)}} \mathrm{F}_{A B}^{C} \quad \text { for all } \mathrm{A} \in \mathrm{S}(\alpha, \mathrm{k}), \\
& \mathrm{n}_{\gamma}(\mathrm{q}) \mathrm{F}_{\alpha \beta}^{\gamma}(\mathrm{q})=\mathrm{n}_{\beta}(\mathrm{q}) \sum_{\substack{\mathrm{A} \in S(\alpha, k) \\
\mathrm{C} \in S(\gamma, k)}} \mathrm{F}_{A B}^{C} \quad \text { for all } \mathrm{B} \in \mathrm{S}(\beta, \mathrm{k}) .
\end{aligned}
$$


Remark 1 The polynomials $\mathrm{F}_{\mathrm{rx}}^{z}$ or $\mathrm{F}_{\mathrm{yr}}^{z}$ where $\mathrm{r}$ is the symbol of a homogeneous regular will denote in our article Hubery's polynomial divided by $\mathrm{n}_{\mathrm{r}}(\mathbf{q})$, which is again a polynomial.

We list now the known tame Hall polynomials associated to indecomposables (see the introduction).

Proposition 1 ([6, 7]) We have the following:

a) Suppose we limit ourselves to defects in $\{-1,0,1\}$. For two roots $x, y$ with $\partial \mathrm{x}=\partial \mathrm{y}=-1$ and $\langle\mathrm{x}, \mathrm{y}\rangle>0$ we have that $\mathrm{F}_{\mathrm{rx}}^{\mathrm{y}}=1$ for any symbol $\mathrm{r}$ corresponding to regular indecomposables of dimension $\mathrm{y}-\mathrm{x}$. This $d u-$ alizes for roots with defect 1 . For roots $x, y$ with $\partial x=-1, \partial y=1$ and $\langle x, y\rangle \neq 0$ we have that $\mathrm{F}_{y x}^{\mathrm{r}}=\frac{1}{\mathrm{q}-1} \alpha_{\mathrm{r}}$ for any symbol $\mathrm{r}$ corresponding to regular indecomposables of dimension $\mathrm{y}-\mathrm{x}$ (where $\alpha_{\mathrm{r}}$ is the number of automorphisms). For three symols corresponding to regular indecomposables the Hall polynomial is classical so it is 0 or 1 . In all the other cases the Hall polynomial is 0 .

b) Let $\mathrm{x}$ be a positive real root with $\partial \mathrm{x}<0$. Then $\mathrm{F}_{\delta-x \mathrm{x}}^{\delta}=\mathrm{h}_{-\partial \mathrm{x}}$, where

$$
\begin{gathered}
h_{1}=1, \\
h_{2}=q-3, \\
h_{3}=q^{2}-5 q+7, \\
h_{4}=q^{3}-6 q^{2}+15 q-14, \\
h_{5}=q^{4}-7 q^{3}+22 q^{2}-37 q+26, \\
h_{6}=q^{5}-7 q^{4}+22 q^{3}-45 q^{2}+62 q-39 .
\end{gathered}
$$

We end this section with a well known lemma:

Lemma 1 Let $\mathrm{P}$ and $\mathrm{P}^{\prime}$ be preprojective indecomposables with $\partial \mathrm{P}=-1$. Then every nonzero morphism $\mathrm{f}: \mathrm{P} \rightarrow \mathrm{P}^{\prime}$ is a monomorphism.

\section{Reductions}

From now on we suppose that $\mathrm{Q}$ is of $\tilde{\mathrm{D}}_{4}$ type.

Our aim is to determine the tame Hall polynomials $\mathrm{F}_{x y}^{z}$ associated to indecomposable modules of defect $\partial z=-2, \partial x=\partial y=-1$ or dually $\partial z=2$, $\partial x=\partial y=1$. 
Using reflection functors (and the fact that Hall numbers are preserved via these functors) one can see that we only need to consider a particularly oriented quiver of $\tilde{D}_{4}$ type (see for example [6] for all the details).

By the arguments above we will consider the quiver $\mathrm{Q}^{\prime}$ of $\tilde{\mathrm{D}}_{4}$ type with all arrows pointing to a non-central vertex (say vertex 1 , the central vertex being $5)$. Thus the unique sink in $Q^{\prime}$ is 1 (one of the marginal vertexes):

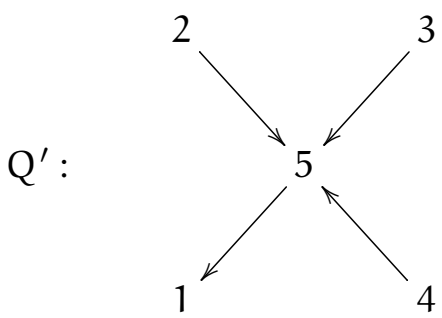

We end this section with the main tool, which will provide us the recursions permitting to compute the Ringel-Hall numbers above.

Proposition 2 [5] Let $\mathrm{X}, \mathrm{Y}, \mathrm{Z} \in \bmod -\mathrm{kQ}$ where $\mathrm{Q}$ is an arbitrary quiver and $\mathrm{k}$ is finite. Denote by $\mathrm{s}_{\mathrm{X}}^{\mathrm{Y}}$ the number of submodules of $\mathrm{Y}$ isomorphic to $\mathrm{X}$, by $\mathrm{f}_{\mathrm{X}}^{\mathrm{Y}}$ the number of submodules of $Y$ with factor isomorphic to $\mathrm{X}$, by $\mathrm{e}_{\mathrm{X}}^{\mathrm{Y}}$ the number of epimorphisms from $\mathrm{Y}$ to $\mathrm{X}$, by $\alpha_{\mathrm{X}}$ the number of automorphisms of $\mathrm{X}$ and by $\mathrm{h}_{\mathrm{XY}}$ the number of morphisms from $\mathrm{X}$ to $\mathrm{Y}$. Then we have the following formula:

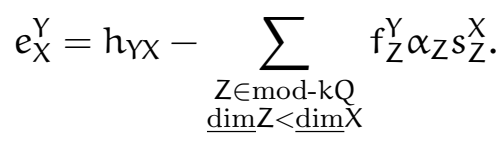

Moreover $e_{X}^{Y}=\alpha_{X} f_{X}^{Y}$.

\section{Recursions and Hall polynomials}

Consider the quiver $\mathrm{Q}^{\prime}$ and the indecomposable preprojectives $\mathrm{P}_{0}, \mathrm{P}^{\prime}, \mathrm{P}$ with $\underline{\operatorname{dim}} \mathrm{P}=\underline{\operatorname{dim}} \mathrm{P}_{0}+\underline{\operatorname{dim}} \mathrm{P}^{\prime}$ and $\partial \mathrm{P}_{0}=\partial \mathrm{P}^{\prime}=-1, \partial \mathrm{P}=-2$. Let $S_{1}$ be the projective simple corresponding to the unique sink 1 . The rest of the indecomposable preprojectives are:

- $P_{2}(n)$ the indecomposable preprojective (of defect -2$)$ with dimension vector $(1,0,0,0,1)+n \delta$; 
- $P_{1}^{1 i}(n)$ (for $i=\overline{1,4}$ ) the indecomposable preprojectives (of defect -1 ) of dimensions $(0,0,0,0,1)+n \delta,(1,1,0,0,1)+n \delta,(1,0,1,0,1)+n \delta$, $(1,0,0,1,1)+n \delta$

- $P_{1}^{2 i}(n)$ (for $i=\overline{1,4}$ ) the indecomposable preprojectives (of defect -1 ) of dimensions $(2,1,1,1,2)+n \delta,(1,0,1,1,2)+n \delta,(1,1,0,1,2)+n \delta$, $(1,1,1,0,2)+n \delta$.

The segment of the preprojective component of the Auslander-Reiten quiver which we will use is the following:
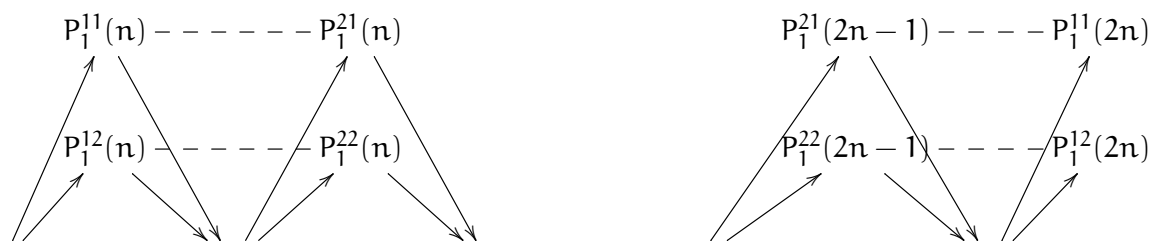

... $\mathrm{P}_{2}(2 \mathrm{n})$

$-P_{2}(2 n+2)$

$\mathrm{P}_{2}(4 \mathrm{n}-1)$
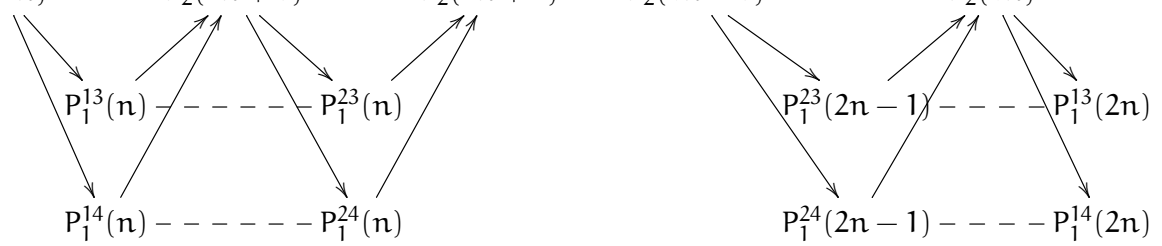

Proposition $3 \mathrm{~F}_{\mathrm{P}^{\prime} \mathrm{P}_{0}}^{\mathrm{P}}=\mathrm{g}_{\mathrm{n}-1}(\mathrm{q})$, where $\mathrm{n}=\left\langle\underline{\operatorname{dim}} \mathrm{P}_{0}, \underline{\operatorname{dim} \mathrm{P}}\right\rangle=\left\langle\underline{\operatorname{dim} P}, \underline{\operatorname{dim}} \mathrm{P}^{\prime}\right\rangle$ and

$$
g_{n}=X^{n}-3 X^{n-1}+\cdots+(-1)^{n-1}(2 n-1) X+(-1)^{n}(n+1)
$$

$\left(\right.$ with $\mathrm{g}_{0}=1$ and $\left.\mathrm{g}_{-1}=0\right)$.

Proof. First of all note that $n=\left\langle\underline{\operatorname{dim}} P_{0}, \underline{\operatorname{dim}} P\right\rangle=\left\langle\underline{\operatorname{dim}} P_{0}, \underline{\operatorname{dim}} P_{0}+\underline{\operatorname{dim}} P^{\prime}\right\rangle=$ $1+\left\langle\underline{\operatorname{dim}} P_{0}, \underline{\operatorname{dim}} P^{\prime}\right\rangle=\left\langle\underline{\operatorname{dim}} P_{0}+\underline{\operatorname{dim}} P^{\prime}, \underline{\operatorname{dim}} P^{\prime}\right\rangle=\left\langle\underline{\operatorname{dim}} P, \underline{\operatorname{dim}} P^{\prime}\right\rangle$. Also if $n=$ $\left\langle\underline{\operatorname{dim}} \mathrm{P}_{0}, \underline{\operatorname{dim}} \mathrm{P}\right\rangle=\left(\mathrm{P}_{0}, \mathrm{P}\right)=0$, then $\mathrm{F}_{\mathrm{P}^{\prime} \mathrm{P}_{0}}^{\mathrm{P}}=0=\mathrm{g}_{-1}$.

We will use induction on $n \geq 1$. For $n=1$, the assertion is trivial since $n=$ $1=\left\langle\underline{\operatorname{dim}} P_{0}, \underline{\operatorname{dim}} \mathrm{P}\right\rangle=\left(\mathrm{P}_{0}, \mathrm{P}\right)$. Using successive Auslander-Reiten translations, the fact that the modules are indecomposable preprojectives and $\underline{\operatorname{dim} P}=$ $\underline{\operatorname{dim}} \mathrm{P}_{0}+\underline{\operatorname{dim}} \mathrm{P}^{\prime}$, one can see that $\mathrm{F}_{\mathrm{P}^{\prime} \mathrm{P}_{0}}^{\mathrm{P}}=\mathrm{F}_{\mathrm{P}_{1} \mathrm{~S}_{1}}^{\mathrm{P}_{2}}$, where $\mathrm{n}=1=\left\langle\underline{\operatorname{dim}} \mathrm{P}_{0}, \underline{\operatorname{dim}} \mathrm{P}\right\rangle=$ $\left\langle\underline{\operatorname{dim}} S_{1}, \underline{\operatorname{dim}} P_{2}\right\rangle=\left(\underline{\operatorname{dim}} P_{2}\right)_{1}$. This means (looking at the dimensions) that $P_{2}=$ $\mathrm{P}_{2}(0)$, and $\mathrm{P}_{1}=\mathrm{P}_{1}^{11}(0)$, so $\mathrm{F}_{\mathrm{P}^{\prime} \mathrm{P}_{0}}^{\mathrm{P}_{0}}=\mathrm{F}_{\mathrm{P}_{1} \mathrm{~S}_{1}}^{\mathrm{P}_{2}}=1$.

Suppose the assertion is true for values under $n$ and prove it for $n$.

Using again successive Auslander-Reiten translations, one can see (as above) that $\mathrm{F}_{\mathrm{P}^{\prime} \mathrm{P}_{0}}^{\mathrm{P}}=\mathrm{F}_{\mathrm{P}_{1} \mathrm{~S}_{1}}^{\mathrm{P}_{2}}$, where $\mathrm{n}=\left\langle\underline{\operatorname{dim}} \mathrm{P}_{0}, \underline{\operatorname{dim} \mathrm{P}}\right\rangle=\left\langle\underline{\operatorname{dim}} S_{1}, \underline{\operatorname{dim}} \mathrm{P}_{2}\right\rangle=\left(\underline{\operatorname{dim}} \mathrm{P}_{2}\right)_{1}$. 
By Proposition 2 we have that

$$
e_{P_{1}}^{P_{2}}=h_{P_{2} P_{1}}-\sum_{\substack{Z \in \bmod -k Q^{\prime} \\ \text { dim } Z<\underline{\text { dim }} P_{1}}} f_{Z_{2}}^{P_{2}} \alpha_{Z} s_{Z}^{P_{1}} .
$$

Note that $F_{P_{1} S_{1}}^{P_{2}}=f_{P_{1}}^{P_{2}}=\frac{e_{P_{1}}^{P_{2}}}{\alpha_{P_{1}}}=\frac{e_{P_{1}}^{P_{2}}}{q-1}$ and $h_{P_{2} P_{1}}=q^{\left(P_{2}, P_{1}\right)}$. Also if there is a monomorphism $Z \rightarrow P_{1}$ and an epimorphism $P_{2} \rightarrow Z$ it follows that $Z=0$ or $Z$ is a indecomposable preprojective of defect -1 such that $\left(Z, P_{1}\right) \neq 0$ and $\left(P_{2}, Z\right) \neq 0$ (here we use the fact that submodules of preprojectives are preprojective and a preprojective of defect -2 can't project on a different preprojective of defect -2$)$. Using the fact that the indecomposable preprojectives are directing, one can see that in the Auslander-Reiten quiver $\mathbf{Z}$ follows after $\mathrm{P}_{2}$ and precedes $\mathrm{P}_{1}$.

Suppose $n=2 m$. Denote by $g_{2 m}^{\prime}=f_{P_{1}^{11}(2 m)}^{P_{2}(2 m)}$.

Using the previous formula and observations and the Auslander-Reiten segment presented above, performing all the calculations we obtain:

$$
\begin{aligned}
g_{2 m}^{\prime} & =f_{P_{1}^{11}(2 m)}^{P_{2}(2 m)} \\
& =\frac{q^{2 m+1}-1}{q-1}-\sum_{\substack{i=\overline{1,4} \\
j=\overline{0, m-1}}} f_{P_{1}^{1 i}(m+j)}^{P_{2}(2 m)} s_{P_{1}^{1 i}(m+j)}^{P_{1}^{11}(2 m)}-\sum_{\substack{i=\overline{1,4} \\
j=\overline{0, m-1}}} f_{P_{1}^{2 i}(m+j)}^{P_{2}(2 m)} s_{P_{1}^{2 i}(m+j)}^{P_{11}^{11}(2 m)}
\end{aligned}
$$

By Lemma 1 we have that

$$
s_{P_{1}^{1 i}(m+j)}^{P_{1}^{11}(2 m)}=\frac{q^{\left(P_{1}^{1 i}(m+j), P_{1}^{11}(2 m)\right)}-1}{q-1}
$$

where $\left(P_{1}^{11}(m+j), P_{1}^{11}(2 m)\right)=m-j+1$ and $\left(P_{1}^{1 i}(m+j), P_{1}^{11}(2 m)\right)=m-j$ for $i=\overline{2,4}$. Also

$$
s_{P_{1}^{2 i}(m+j)}^{P_{1}^{11}(2 m)}=\frac{q^{\left(P_{1}^{2 i}(m+j), P_{1}^{11}(2 m)\right)}-1}{q-1}
$$

where $\left(P_{1}^{21}(m+j), P_{1}^{11}(2 m)\right)=m-j-1$ and $\left(P_{1}^{2 i}(m+j), P_{1}^{11}(2 m)\right)=m-j$ for $i=\overline{2,4}$.

The kernel of an epimorphism $P_{2}(2 m) \rightarrow P_{1}^{l i}(m+j)$ is preprojective and of defect -1 , so it is indecomposable and unique. Denote it by $X$. This implies that $f_{P_{1}^{l i}(m+j)}^{P_{2}(2 m)}=F_{P_{1}^{l i}(m+j) X}^{P_{2}(2 m)}$. Using the induction hypothesis one can deduce that $F_{P_{1}^{1 i}(m+j) X}^{P_{2}(2 m)}=g_{2 j}(q)$ and $F_{P_{1}^{2 i}(m+j) X}^{P_{2}(2 m)}=g_{2 j+1}(q)$, since $\left\langle P_{2}(2 m), P_{1}^{1 i}(m+j)\right\rangle=$ $2 j+1$ and $\left\langle P_{2}(2 m), P_{1}^{2 i}(m+j)\right\rangle=2 j+2$. 
Substituting everything in (1) we obtain:

$$
\begin{aligned}
g_{2 m}^{\prime} & =\frac{q^{2 m+1}-1}{q-1}-\frac{q^{m+1}-1}{q-1} g_{0}(q) \\
& -\sum_{j=1, m-1} \frac{q^{m-j+1}-1}{q-1}\left(g_{2 j}(q)+3 g_{2 j-1}(q)+3 g_{2 j-2}(q)+g_{2 j-3}(q)\right) \\
& -3 g_{2 m-1}(q)-3 g_{2 m-2}(q)-g_{2 m-3}(q) .
\end{aligned}
$$

In case $n=2 m+1$ a similar recursion can be obtained for $g_{2 m+1}^{\prime}$. More precisely we get:

$$
\begin{aligned}
g_{2 m+1}^{\prime} & =\frac{q^{2 m+2}-1}{q-1}-\frac{q^{m+1}-1}{q-1}\left(g_{1}(q)+3 g_{0}(q)\right) \\
& -\sum_{j=\frac{1, m-1}{q}} \frac{q^{m-j+1}-1}{q-1}\left(g_{2 j+1}(q)+3 g_{2 j}(q)+3 g_{2 j-1}(q)+g_{2 j-2}(q)\right) \\
& -3 g_{2 m}(q)-3 g_{2 m-1}(q)-g_{2 m-2}(q) .
\end{aligned}
$$

By direct calculation we get that $g_{2 m}^{\prime}=g_{2 m}(q)$ and $g_{2 m+1}^{\prime}=g_{2 m+1}(q)$ that is, $g_{n}^{\prime}=g_{n}(q)$ for all $n$.

Remark 2 Based on calculations done with a computer we conjecture that the polynomials above are irreducible (as integer polynomials).

\section{Acknowledgment}

Supported by a grant of the Babeș-Bolyai University (GTC 2019-2020).

\section{References}

[1] I. Assem, D. Simson, A. Skowronski, Elements of Representation Theory of Associative Algebras, Volume 1: Techniques of Representation Theory, LMS Student Texts (No. 65), Cambridge Univ. Press 2006.

[2] M. Auslander, I. Reiten, S. Smalo, Representation Theory of Artin Algebras, Cambridge Stud. in Adv. Math. 36, Cambridge Univ. Press 1995.

[3] V. Dlab, C. M. Ringel, Indecomposable representations of graphs and algebras, AMS Memoirs 173, 1976. 
[4] A. Hubery, Hall polynomials for affine quivers, Represent. Theory, 14 (2010), 355-378.

[5] C. M. Ringel, Hall algebras, in: Topics in algebra, Banach Center Publ., 26 (1990), 433-447.

[6] Cs. Szántó, On some Ringel-Hall products in tame cases, Journal of Pure and Applied Algebra, 216 (2012), 2069-2078.

[7] Cs. Szántó, I. Szöllősi, Hall polynomials and the Gabriel-Roiter submodules of simple homogeneous modules, Bulletin of the London Mathematical Society, 47 (2), (2015), 206-216.

Received: October 27, 2020 